\title{
Descriptor realizations of autoregressive representations
}

\author{
N. P. KARAMPETAKIS $\dagger$ \\ Department of Mathematics, Aristotle University of Thessaloniki, Thessaloniki 54006, \\ Greece
}

[Received on 28 February 2003]

\begin{abstract}
In Pugh et al. (1998, Automatica, submitted), fundamental equivalence between AutoRegressive (AR) representations has been defined. Actually, two AR-representations are defined to be fundamentally equivalent in the case there exists a bijective polynomial differential map between their behaviours. In this paper, it is shown how to reduce an AR-representation to a fundamental equivalent realization in descriptor form.
\end{abstract}

Keywords: autoregressive representations; descriptor systems; equivalence; realization; full equivalence.

\section{Introduction}

The problem of equivalence between AutoRegressive (AR) representations has been the concern of many scientists in recent years. While the equivalence between regular polynomial descriptions (PMDs) has been extensively studied e.g. Rosenbrock (1970) and Hayton et al. (1988), the same has not happened in the case of non-regular ARRepresentations. There are essentially two main approaches to the solution of this problem. The first one gives characterization of equivalence in terms of preservation of the structural invariants of the matrices describing the systems, while the second deals with the relations of the solution sets or behaviours of the corresponding systems.

The most known transformation between AR-representations is the external equivalence. Systems are called externally equivalent if their induced smooth behaviours are the same. For more details and motivation the reader is referred to Kuijper (1994). Moreover, it is shown in Kuijper (1994) that in case where two systems are externally equivalent, then the polynomial matrices describing the two systems are unimodular equivalent and, therefore, have the same finite zero structure. In order to treat both the smooth and impulsive behaviour in the same manner, (Pugh et al., 1998) define the notion of fundamental equivalence between AR-representations. Systems are called fundamentally equivalent if their induced smooth and impulsive behaviours are connected through a bijective polynomial differential map. It is shown in Pugh et al. (1998) that in the case where two systems are fundamentally equivalent, then the polynomial matrices describing the two systems are fully equivalent and, therefore, have the same finite and infinite zero structure. The only disadvantage of the theory of fundamental equivalence is that it applies, until now, only to systems which are described by full row rank polynomial matrices. Although this does not matter if we are interested in the smooth behaviour of the system (Kuijper, 1994), it plays a role as concerns the admissible initial conditions of the

\footnotetext{
†Email: karampet@math.auth.gr
} 
systems when we are interested both in the smooth and impulsive behaviour of systems (Karampetakis \& Vardulakis, 1993).

One of the interesting applications of the above transformations is the reduction of an AR-Representation which is described by a polynomial matrix of order greater than one, to an equivalent AR-representation in pencil or descriptor form. In the case where we are only interested in the smooth behaviour, a reduction method has already been proposed by Kuijper \& Shumacher (1990) and Kuijper (1994) using the external equivalence transformation.

In this paper we propose a new reduction method, using the trasformation of fundamental equivalence, which has the nice property of preserving both the smooth and impulsive behaviour of the equivalent systems. More specifically, in Section 2 we present some preliminary results concerning the definition of (a) the behaviour of AR-representations and (b) the fundamental equivalence between AR-representations. In Section 3 we formulate our problem while in Section 4 we propose an algebraic algorithm which reduces a full row rank AR-representation to a fundamental equivalent AR-representation in pencil form. The above algorithm is illustrated via an example in Section 5 while it is classified to a more theoretical algorithm presented later in Section 6.

\section{Preliminary results}

Consider an AR-representation described by

$$
\Sigma_{R}: R(\rho) w(t)=0
$$

where $\rho:=\mathrm{d} / \mathrm{d} t$ is the differential operator, $R(\rho)=R_{q} \rho^{q}+R_{q-1} \rho^{q-1}+\cdots+R_{1} \rho+R_{0} \in$ $R[\rho]^{p \times m}$ and $w(t) \in D_{+}^{\prime m}$ is vector valued distribution of appropriate dimension. Now by applying Laplace transforms on (1) we have

$$
R(s) w(s)=S_{q-1} X_{R} \bar{w}(0-)
$$

where

$$
\begin{gathered}
S_{q-1}=\left[\begin{array}{llll}
s^{q-1} I_{p} & \cdots & s I_{p} & I_{p}
\end{array}\right] \\
\bar{w}(0-)=\left[\begin{array}{c}
w(0-) \\
w^{(1)}(0-) \\
\vdots \\
w^{(q-1)}(0-)
\end{array}\right] \\
X_{R}=\left[\begin{array}{cccc}
R_{q} & 0 & \cdots & 0 \\
R_{q-1} & R_{q} & \cdots & 0 \\
\vdots & \vdots & \ddots & \vdots \\
R_{1} & R_{2} & \cdots & R_{q}
\end{array}\right] .
\end{gathered}
$$

The vectors $w^{(i)}(0-)$ will be termed the initial values of $w(t)$, while $X_{R} \bar{w}(0-)$ will be termed the initial conditions of $w(t)$. It is easily seen from (2) that the initial conditions 
$X_{R} \bar{w}(0-)$ uniquely specify the right-hand side of equation (2) because $S_{q-1}$ is always an isomorphism. It is known (Karampetakis \& Vardulakis, 1993) that the AR-representation (1) has a solution if certain constraints between the initial conditions are satisfied. The system is solvable for every initial condition (Pugh et al., 1998) iff all the left minimal indices of $R(s)$ are zero. In that case it can be reduced to an equivalent full row rank system, with the same solution space. It is clear that the full row rank assumption can be made without loss of generality for systems that are solvable for every initial condition. Thus, we can restrict what follows to the full row rank case, i.e. we shall assume that $\operatorname{rank} R(s)=p$.

Following the terminology of Willems (1991) we denote by $B_{R}$ the trajectory or behaviour of $\Sigma_{R}$, i.e.

$$
B_{R}:=\left\{w(t) \in D_{+}^{\prime m}: R(\rho) w(t)=0, t \in[0-,+\infty)\right\} .
$$

In the case where $R(s)$ is square and non-singular, $B_{R}$ is a finite-dimensional space with dimension equal to the sum of the finite and infinite zeros of $R(s)$ (Vardulakis, 1991), while in the case where $R(s)$ is not square, then $B_{R}$ is an infinite-dimensional vector space.

An alternative aproach to the question of what constitutes the solution of non-regular AR-representations, which overcomes this problem, has been proposed in Karampetakis \& Vardulakis (1993). According to this approach, the trajectory space $B_{R}$ can be partitioned according to the equivalence relation

$$
w(t) \sim w(t)^{\prime} \Leftrightarrow X_{R} \bar{w}(0-)=X_{R} \bar{w}^{\prime}(0-) .
$$

It is easily seen that the above equivalence relation seperates the behaviour space $B_{R}$ into equivalence classes where each equivalence class $[w(t)]$ consists of solutions of (1) having the same initial conditions. Thus, if we define

$$
Z_{R}=\left\{w(t) \in B_{R}: X_{R} \bar{w}(0-)=0\right\}
$$

then each equivalence class of $w(t) \in B_{R}$ can be rewritten as

$$
[w(t)]=w(t)+Z_{R} .
$$

It seems quite natural to introduce the notion of the quotient solution space of (1) as follows.

DEFINITION 1 (Karampetakis \& Vardulakis, 1993) The quotient solution space of (1) is defined as

$$
\bar{B}_{R}=B_{R} / Z_{R} .
$$

According to the above definition there is one to one correspodence between the initial condition vectors $X_{R} \bar{w}(0-)$ and the elements of $\bar{B}_{R}$ and thus an isomorphism between the initial condition space and the quotient solution space. An interesting theorem concerning the dimension of $\bar{B}_{R}$ is given below.

THEOREM 2 (Karampetakis \& Vardulakis, 1993) The quotient solution space $\bar{B}_{R}$ has dimension

$$
\operatorname{dim} \bar{B}_{R}=n+q+\varepsilon
$$


where $n, q, \varepsilon$ denotes respectively the total number of finite zeros, infinite zeros and right minimal indices (order accounted for).

On this basis the quotient solution space is a finite-dimensional view of the solution space of AR-representation (1) while the behaviour is an infinite-dimensional and complete view of the solution space.

Let now $\Sigma_{1}, \Sigma_{2}$ be two AR-Representations described by the following equations:

$$
\Sigma_{i}: R_{i}(\rho) w_{i}(t)=0 ; R_{i}(\rho) \in R[\rho]^{p_{i} \times m_{i}} i=1,2
$$

with

$$
B_{i}=\left\{w_{i}(t) \in D_{+}^{\prime m_{i}}: R_{i}(\rho) w_{i}(t)=0 ; i=1,2\right\}
$$

Then fundamental equivalence between $\Sigma_{1}, \Sigma_{2}$ can be defined as follows.

Definition 3 (Pugh et al., 1998) The systems described by (5) are fundamentally equivalent iff there exists a bijective polynomial differential map $N(\rho): B_{1} \rightarrow B_{2}$.

In the case where this map exists, then it was shown in Pugh et al. (1998) that one can induce naturally a unique well-defined map between the quotient solution spaces of $\Sigma_{1}, \Sigma_{2}$. The fundamental equivalence transformation is related to the full equivalent transformation according to the following theorem.

THEOREM 4 (Pugh et al., 1998) The systems described by the AR-representations (5) are fundamentally equivalent iff the polynomial matrices $R_{1}(s), R_{2}(s)$ are fully equivalent i.e. $\exists$ polynomial matrices $M(s) \in R[s]^{p_{2} \times p_{1}}$ and $N(s) \in R[s]^{m_{2} \times m_{1}}$ satisfying the following conditions:

$$
\left[M(s) \quad R_{2}(s)\right]\left[\begin{array}{c}
R_{1}(s) \\
-N(s)
\end{array}\right]=0
$$

where the compound matrices

$$
\left[M(s) \quad R_{2}(s)\right] ;\left[\begin{array}{c}
R_{1}(s) \\
-N(s)
\end{array}\right]
$$

(i) have full normal rank,

(ii) have no finite nor infinite zeros,

(iii)

$$
\begin{gathered}
\delta_{M}\left[M(s) \quad R_{2}(s)\right]=\delta_{M}\left[R_{2}(s)\right] \\
\delta_{M}\left[\begin{array}{c}
R_{1}(s) \\
-N(s)
\end{array}\right]=\delta_{M}\left[R_{1}(s)\right],
\end{gathered}
$$

(iv) $m_{1}-p_{1}=m_{2}-p_{2}$.

In that case the systems described by (5) are connected through the bijective polynomial differential map $N(\rho): B_{1} \rightarrow B_{2}$.

It is obvious from above that fundamental equivalence is a behavioural interpretation of full matrix equivalence and, subsequently, a connection between the behavioural approach and the theory of polynomial matrix transformations. 


\section{Problem formulation}

Given the AR-Representation of $\Sigma_{1}$

$$
\begin{gathered}
\Sigma_{1}: R(\rho) w(t)=0 \\
B_{1}:=\{w(t): R(\rho) w(t)=0, t \in[0,+\infty]\}
\end{gathered}
$$

determine an AR-Representation in pencil form

$$
\begin{gathered}
\Sigma_{2}:(\rho E-A) \xi(t)=0 \\
B_{2}:=\{\xi(t):(\rho E-A) \xi(t)=0, t \in[0,+\infty]\}
\end{gathered}
$$

which is fundamentally equivalent to (6), i.e. there exists a bijective polynomial differential map

$$
N: \xi(t) \in B_{1} \rightarrow N(\rho) \xi(t) \in B_{2}
$$

between the induced smooth and impulsive behaviours of $\Sigma_{1}$ and $\Sigma_{2}$.

\section{Realization procedure}

The realization procedure described in this section is based on Tan \& Vandewall's (1998) algorithm for the generalized state space realization of matrix fraction descriptions.

Algorithm 1 The realization $\left[s E-A \in R[s]^{l_{1} \times l_{2}}, B \in R^{l_{2} \times m}, C \in R^{p \times l_{1}}\right]$ defined by the following procedure is a generalized state space realization of $R(s) \in R[s]^{p \times m}$.

Step 1. Find a unimodular matrix $U(s) \in R[s]^{p \times p}$ which transforms the compound matrix $\left[\begin{array}{ll}R(s) & I_{p}\end{array}\right]$ to a row reduced form, i.e. such that the matrix:

$$
[N(s) \quad D(s)]=[U(s) R(s) \quad U(s)]
$$

is row reduced.

Step 2. Let

$$
\left.S_{[N}^{\infty} \quad D\right]^{(s)}=\left[\operatorname{diag}\left[\begin{array}{lllll}
s^{q_{1}} & s^{q_{2}} & \cdots & s^{q_{p}}
\end{array}\right] 0_{p m}\right]
$$

be the Smith form at $s=\infty$ of the compound matrix (8), where $q_{1} \geqslant q_{2} \geqslant \cdots \geqslant q_{p} \geqslant 0$ are the orders of the infinite poles of this compound matrix which coincides with the row degrees of the same compound matrix because of row reducedness (Vardulakis, 1991). Define the matrix

$$
S(s)=\operatorname{blockdiag}\left[\left[\begin{array}{llll}
s^{q_{1}} & s^{q_{2}} & \ldots & 1
\end{array}\right] i=1,2, \ldots, p\right]
$$

and write the polynomial matrices $N(s)$ and $D(s)$ as follows:

$$
N(s)=S(s) N_{C} ; D(s)=S(s) D_{C} .
$$


Step 3. Construct the core realization

$$
s E_{C}-A_{C}=\text { blockdiag }\left[s E_{C_{1}}-A_{C_{1}} \quad \cdots \quad s E_{C_{p}}-A_{C_{p}}\right]
$$

where

$$
s E_{C_{i}}-A_{C_{i}}=\left[\begin{array}{cccc}
0 & -1 & \cdots & 0 \\
0 & s & \cdots & 0 \\
\vdots & \vdots & \ddots & \vdots \\
0 & 0 & \cdots & -1 \\
0 & 0 & \cdots & s
\end{array}\right] \in R[s]^{\left(q_{i}+1\right) \times\left(q_{i}+1\right)} i=1,2, \ldots, p
$$

and

$$
\begin{gathered}
C_{C}=\text { blockdiag }\left[C_{1}, \quad C_{2}, \cdots \quad C_{p}\right] \\
C_{i}=\left[\begin{array}{cccc}
1 & 0 & \cdots & 0
\end{array}\right] \in R^{1 \times\left(q_{i}+1\right)} \\
B_{C}=I_{n} n=\sum_{i=1}^{p} q_{i}+p .
\end{gathered}
$$

Step 4. Define the matrices

$$
E=E_{C} ; A=A_{C}-D_{C} C_{C} ; B=N_{C} ; C=C_{C} .
$$

Proof. It is easily checked that

$$
S(s)(s E-A)=S(s) D_{C} C=D(s) C
$$

or equivalently

$$
C(s E-A)^{-1}=D(s)^{-1} S(s) .
$$
that

By postmultiplying with $B\left(=N_{C}\right)$ and by observing that $N(s)=S(s) N_{C}$ we have

$$
C(s E-A)^{-1} B=D(s)^{-1} N(s)=R(s) .
$$

An interesting lemma concerning the McMillan degree of a coprime at $\mathrm{C} \cup\{\infty\}$ matrix fraction description is given in the sequel.

LEMMA 5 (Janssen, 1988) Let $T(s) \in R(s)^{p \times m}$ and $T(s)=Q(s)^{-1} R(s)(=$ $\left.R(s) Q(s)^{-1}\right), R(s) \in R[s]^{p \times m}, Q(s) \in R[s]^{p \times p},\left(Q(s) \in R[s]^{m \times m}\right)$, is a left (right) matrix fraction description of $T(s)$. If the compound matrix $\left[\begin{array}{lll}Q(s) & R(s)\end{array}\right]$ $\left(\left[\begin{array}{ll}R(s) & Q(s)\end{array}\right]^{T}\right.$ ) has no zeros in $C \cup\{\infty\}$ then

$$
\delta_{M}\left(Q(s)^{-1} R(s)\right)=\sum_{i=1}^{k} q_{i}\left[\begin{array}{ll}
Q & R
\end{array}\right]=\delta_{M}[Q(s) \quad R(s)]
$$

where $q_{i}\left[\begin{array}{ll}Q & R\end{array}\right]>0$ are the orders of the infinite poles of $\left[\begin{array}{ll}Q(s) & R(s)\end{array}\right]$. 
Define now the pencil

$$
s \bar{E}-\bar{A}=\left[\begin{array}{cc}
s E-A & B \\
-C & 0
\end{array}\right] \in R^{\bar{l}_{1} \times \bar{l}_{2}}
$$

where

$$
\begin{gathered}
\bar{l}_{1} \times \bar{l}_{2}=\left(p+\sum_{i=1}^{p}\left(q_{i}\left[\begin{array}{ll}
D^{\mathrm{T}} & N^{\mathrm{T}}
\end{array}\right]^{\mathrm{T}}+1\right)\right) \\
\times\left(m+\sum_{i=1}^{p}\left(q_{i}\left[\begin{array}{ll}
D^{\mathrm{T}} & N^{\mathrm{T}}
\end{array}\right]^{\mathrm{T}}+1\right)\right) \\
\stackrel{\text { Lemma } 5}{=}\left(2 p+\delta_{M}(R)\right) \times\left(p+m+\delta_{M}(R)\right)
\end{gathered}
$$

Then we have the following theorem.

THEOREM 6 The polynomial matrix $R(s)$ and the pencil $s \bar{E}-\bar{A}$ are fully equivalent under the following full equivalent transformations:

$$
\begin{aligned}
& {\left[D(s)^{-1} S(s) \quad I\right][s \bar{E}-\bar{A}]=R(s)\left[\begin{array}{ll}
0 & I_{m}
\end{array}\right]} \\
& {\left[\begin{array}{c}
0 \\
I_{p}
\end{array}\right] R(s)=[s \bar{E}-\bar{A}]\left[\begin{array}{c}
-\left[\begin{array}{c}
s E-A]^{-1} \\
I_{n}
\end{array}\right] .
\end{array}\right.}
\end{aligned}
$$

Proof. In order to prove this theorem we must show that the conditions (i)-(iv) defined in Theorem 4 are satisfied. Consider the transformation (11) or equivalently

$$
\left[D(s)^{-1} S(s) \quad I \quad R(s)\right]\left[\begin{array}{cc}
s E-A & B \\
-C & 0 \\
0 & -I_{m}
\end{array}\right]=0 .
$$

(a) The compound matrix

$$
M(s)=\left[\begin{array}{cc}
s E-A & B \\
-C & 0 \\
0 & -I_{m}
\end{array}\right]
$$

is strongly equivalent (Verghese et al., 1981) to the matrix

$$
\begin{gathered}
{\left[\begin{array}{cc}
s E_{C}-A_{C} & 0 \\
-C_{C} & 0 \\
0 & -I_{m}
\end{array}\right]} \\
=\left[\begin{array}{ccc}
I & D_{C} & N_{C} \\
0 & I & 0 \\
0 & 0 & I
\end{array}\right]\left[\begin{array}{cc}
s E-A & B \\
-C & 0 \\
0 & -I_{m}
\end{array}\right]
\end{gathered}
$$

which has no zeros at $C \cup\{\infty\}$ because of its special structure (see (9)-(10)). The McMillan degree conditions are also satisfied because the constant terms $\left(\left[\begin{array}{ll}0 & -I_{m}\end{array}\right]\right)$ play no role in the McMillan degree of a polynomial matrix (Pugh, 1976). 
(b) Consider now the second compound matrix

$$
Q(s)=\left[D(s)^{-1} S(s) \quad I \quad R(s)\right]
$$

We observe that

$$
\left[D(s)^{-1} S(s) \quad I \quad R(s)\right]=D(s)^{-1}\left[\begin{array}{lll}
S(s) & D(s) & N(s)
\end{array}\right]
$$

is a left coprime matrix fraction description, i.e. the compound matrix $\left[\begin{array}{lll}D(s) & N(s)\end{array}\right]$ has no finite zeros. It is easily seen, because of the special structure of $S(s)$, that the compound matrix $\left[\begin{array}{llll}S(s) & D(s) & N(s) & D(s)\end{array}\right]$ is row reduced and thus has no infinite zeros. Thus, we conclude that $Q(s)$ has no zeros at $C \cup\{\infty\}$.

$\left[\begin{array}{llll}S(s) & D(s) & N(s) & D(s)\end{array}\right]$ is row reduced and thus from Lemma 5 we have that

$$
\begin{aligned}
& \delta_{M}\left[\begin{array}{lll}
D(s)^{-1} S(s) & I & R(s)
\end{array}\right]=\delta_{M}\left[\begin{array}{llll}
S & D & N & D
\end{array}\right] \\
= & \sum_{i=1}^{m} q_{i}\left[\begin{array}{ll}
D(s) & N(s)]=\delta_{M}\left(D(s)^{-1} N(s)\right)=\delta_{M}(R(s))
\end{array}\right.
\end{aligned}
$$

which verifies the McMillan degree condition of the compound matrix $Q(s)$.

(c) We observe also that

$$
\bar{l}_{1}-\bar{l}_{2}=\left(2 p+\delta_{M}(R)\right)-\left(p+m+\delta_{M}(R)\right)=p-m
$$

From (a)-(c) we conclude that (11) is a full equivalent transformation. In a similar way we can prove that (12) is also a full equivalent transformation.

Using now Theorem 6 we can easily prove the following theorem.

THEOREM 7 The AR-representations

$$
\Sigma_{1}: R(\rho) w(t)=0 \text { and } \Sigma_{2}:(\rho \bar{E}-\bar{A}) \xi(t)=0
$$

are fundamentally equivalent.

Proof. The polynomial matrices $R(s),(s \bar{E}-\bar{A})$ are fully equivalent according to Theorem 6, and related through the full equivalent transformations (11) and (12). Thus $\Sigma_{1}$ and $\Sigma_{2}$ are fundamentally equivalent according to Theorem 4 . The bijective polynomial differential maps which relate the behavioural spaces of $\Sigma_{1}$ and $\Sigma_{2}$ are coming from the right transforming matrices in relations (11) and (12) and are the following:

$$
w(t)=\left[\begin{array}{cc}
0 & I_{m}
\end{array}\right] \xi(t) ; \xi(t)=\left[\begin{array}{c}
-[\rho E-A]^{-1} B \\
I_{m}
\end{array}\right] w(t) .
$$




\section{Illustrative example}

Consider the AR-Representation

$$
\underbrace{\left(\begin{array}{ccc}
\rho^{3}+\rho^{2} & \rho^{3}+\rho^{2}-1 & \rho^{3}-\rho \\
-\rho^{2}-\rho & -\rho^{2}-\rho & -\rho^{2}+1
\end{array}\right)}_{R(\rho)} \underbrace{\left(\begin{array}{l}
w_{1}(t) \\
w_{2}(t) \\
w_{3}(t)
\end{array}\right)}_{w(t)}=0
$$

where $R(\rho)$ has full row rank and

$$
\begin{gathered}
S_{R(s)}^{C}(s)=\left(\begin{array}{ccc}
1 & 0 & 0 \\
0 & s+1 & 0
\end{array}\right) ; S_{R(s)}^{\infty}(s)=\left(\begin{array}{ccc}
s^{3} & 0 & 0 \\
0 & \frac{1}{s} & 0
\end{array}\right) \\
\left(\begin{array}{ccc}
s^{3}+s^{2} & s^{3}+s^{2}-1 & s^{3}-s \\
-s^{2}-s & -s^{2}-s & -s^{2}+1
\end{array}\right)\left(\begin{array}{c}
-s+1 \\
0 \\
s
\end{array}\right)=0 .
\end{gathered}
$$

Applying Algorithm 1 we have the following steps.

Step 1. There is a unimodular matrix

$$
U(s):=\left(\begin{array}{cc}
1 & s \\
0 & 1
\end{array}\right) \in R[s]^{2 \times 2}
$$

such that

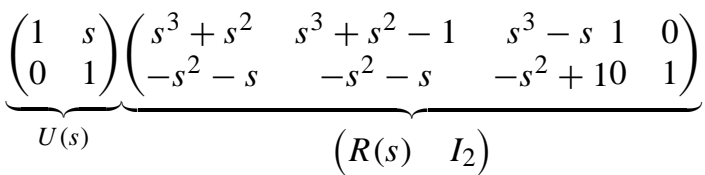

$$
\begin{aligned}
& =\left(\begin{array}{ccccc}
0 & -1 & 0 & 1 & s \\
-s^{2}-s & -s^{2}-s & -s^{2}+1 & 0 & 1 \\
N(s) & & \underbrace{}_{D(s)}
\end{array}\right)
\end{aligned}
$$

is row reduced.

Step 2. Define the matrix

$$
S(s)=\left(\begin{array}{ccccc}
s & 1 & 0 & 0 & 0 \\
0 & 0 & s^{2} & s & 1
\end{array}\right)
$$

and write the polynomial matrices $N(s)$ and $D(s)$ as follows:

$$
N(s)=\underbrace{\left(\begin{array}{ccccc}
s & 1 & 0 & 0 & 0 \\
0 & 0 & s^{2} & s & 1
\end{array}\right)}_{S(s)} \underbrace{\left(\begin{array}{ccc}
0 & 0 & 0 \\
0 & -1 & 0 \\
-1 & -1 & -1 \\
-1 & -1 & 0 \\
0 & 0 & 1
\end{array}\right)}_{N_{C}}
$$




$$
D(s)=\underbrace{\left(\begin{array}{ccccc}
s & 1 & 0 & 0 & 0 \\
0 & 0 & s^{2} & s & 1
\end{array}\right)}_{S(s)} \underbrace{\left(\begin{array}{ll}
0 & 1 \\
1 & 0 \\
0 & 0 \\
0 & 0 \\
0 & 1
\end{array}\right)}_{D_{C}} .
$$

Step 3. Construct the core realization

$$
s E_{C}-A_{C}=\left(\begin{array}{ccccc}
0 & -1 & 0 & 0 & 0 \\
0 & s & 0 & 0 & 0 \\
0 & 0 & 0 & -1 & 0 \\
0 & 0 & 0 & s & -1 \\
0 & 0 & 0 & 0 & s
\end{array}\right)
$$

and

$$
C_{C}=\left(\begin{array}{ccccc}
1 & 0 & 0 & 0 & 0 \\
0 & 0 & 1 & 0 & 0
\end{array}\right) ; B_{C}=I_{5} .
$$

Step 4. Define the matrices

$$
\begin{aligned}
E=E_{C}= & \left(\begin{array}{ccccc}
0 & 0 & 0 & 0 & 0 \\
0 & 1 & 0 & 0 & 0 \\
0 & 0 & 0 & 0 & 0 \\
0 & 0 & 0 & 1 & 0 \\
0 & 0 & 0 & 0 & 1
\end{array}\right) \\
A=A_{C}-D_{C} C_{C} & =\left(\begin{array}{ccccc}
0 & 1 & -1 & 0 & 0 \\
-1 & 0 & 0 & 0 & 0 \\
0 & 0 & 0 & 1 & 0 \\
0 & 0 & 0 & 0 & 1 \\
0 & 0 & -1 & 0 & 0
\end{array}\right) \\
B & =N_{C}=\left(\begin{array}{ccc}
0 & 0 & 0 \\
0 & -1 & 0 \\
-1 & -1 & -1 \\
-1 & -1 & 0 \\
0 & 0 & 1
\end{array}\right) \\
C & =C_{C}=\left(\begin{array}{ccccc}
1 & 0 & 0 & 0 & 0 \\
0 & 0 & 1 & 0 & 0
\end{array}\right) .
\end{aligned}
$$

Thus the generalized state space system

$$
\left(\begin{array}{cc}
\rho E-A & B \\
-C & 0
\end{array}\right) \xi(t)=0 \Leftrightarrow
$$




$$
\left(\begin{array}{cccccccc}
0 & -1 & 1 & 0 & 0 & 0 & 0 & 0 \\
1 & \rho & 0 & 0 & 0 & 0 & -1 & 0 \\
0 & 0 & 0 & -1 & 0 & -1 & -1 & -1 \\
0 & 0 & 0 & \rho & -1 & -1 & -1 & 0 \\
0 & 0 & 1 & 0 & \rho & 0 & 0 & 1 \\
-1 & 0 & 0 & 0 & 0 & 0 & 0 & 0 \\
0 & 0 & -1 & 0 & 0 & 0 & 0 & 0
\end{array}\right) \xi(t)=0
$$

is fundamentally equivalent to the AR-Representation (13). The bijective polynomial differential map which connects the behavioural spaces of the two systems is the following:

$$
\begin{gathered}
w(t)=\left[\begin{array}{ll}
0_{3,5} & I_{3}
\end{array}\right] \xi(t) \\
\xi(t)=\left[\begin{array}{cc}
-[\rho E-A]^{-1} B \\
I_{3}
\end{array}\right] w(t) \Leftrightarrow \\
\xi(t)=\left(\begin{array}{ccc}
-\rho^{3}-\rho^{2} & 1-\rho^{3}-\rho^{2} & -\rho^{3}+\rho \\
\rho^{2}+\rho & \rho^{2}+\rho & \rho^{2}-1 \\
\rho^{2}+\rho & \rho^{2}+\rho & \rho^{2}-1 \\
-1 & -1 & -1 \\
-\rho-1 & -\rho-1 & -\rho \\
1 & 0 & 0 \\
0 & 1 & 0 \\
0 & 0 & 1
\end{array}\right) w(t) .
\end{gathered}
$$

\section{An extension of the realization procedure}

The main aim of this section is to classify the reduction method presented in the previous section, to a new reduction theoretical model. In order to present this new method we first need to present the following lemma.

LEMMA 8 (Karampetakis \& Vardulakis, 1992) Let

$$
P_{i}(s)=\left(\begin{array}{cc}
A_{i}(s) & B_{i}(s) \\
-C_{i}(s) & D_{i}(s)
\end{array}\right) i=1,2
$$

be the Rosenbrock system matrices of two strongly irreducible systems $\Sigma_{i} i=1$, 2, i.e. the compound matrices

$$
\left(\begin{array}{ccc}
A_{i}(s) & B_{i}(s) & 0 \\
-C_{i}(s) & D_{i}(s) & I
\end{array}\right) \text { and }\left(\begin{array}{cc}
A_{i}(s) & B_{i}(s) \\
-C_{i}(s) & D_{i}(s) \\
0 & I
\end{array}\right)
$$

have no finite or infinite zeros (Verghese et al., 1981). Then the two systems have the same transfer function, i.e. $G_{1}(s)=G_{2}(s)$ where $G_{i}(s)=C_{i}(s) A_{i}(s)^{-1} B_{i}(s)+D_{i}(s)$ iff

$$
\exists M^{\prime}(s)=\left(\begin{array}{cc}
M(s) & 0 \\
X(s) & I
\end{array}\right) \text { and } N^{\prime}(s)=\left(\begin{array}{cc}
N(s) & Y(s) \\
0 & I
\end{array}\right)
$$

such that

$$
M^{\prime}(s) P_{1}(s)=P_{2}(s) N^{\prime}(s)
$$

is a full equivalent transformation. 
Now we can easily show the following theorem.

THEOREM 9 Let $\Sigma_{R}$ be the system described by the AR-Representation (1). Let also $\Sigma_{G S}$ be the system described by the following AR-Representation:

$$
\Sigma_{G S}:\left(\begin{array}{cc}
A_{0}(\rho) & B_{0}(\rho) \\
-C_{0}(\rho) & D_{0}(\rho)
\end{array}\right) \xi(t)=0
$$

where $\left\{A_{0}(s), B_{0}(s), C_{0}(s), D_{0}(s)\right\}$ is a strongly irreducible realization of $R(s)$ formed by polynomial matrices in pencil form, i.e.

$$
R(s)=C_{0}(s) A_{0}(s)^{-1} B_{0}(s)+D_{0}(s) .
$$

Then $\Sigma_{R}$ and $\Sigma_{G S}$ are fundamentally equivalent.

Proof. The Rosenbrock system matrices

$$
P_{1}(s)=\left(\begin{array}{cc}
I & 0 \\
0 & R(s)
\end{array}\right) ; P_{2}(s)=\left(\begin{array}{cc}
A_{0}(s) & B_{0}(s) \\
-C_{0}(s) & D_{0}(s)
\end{array}\right)
$$

define two strongly irreducible systems, $\Sigma_{1}$ and $\Sigma_{2}$ respectively, with the same transfer function, i.e. $R(s)$. Thus, according to Lemma $8, \exists M(s), N(s), X(s), Y(s)$ such that

$$
\begin{gathered}
\left(\begin{array}{cc}
M(s) & 0 \\
X(s) & I
\end{array}\right)\left(\begin{array}{cc}
I & 0 \\
0 & R(s)
\end{array}\right) \\
=\left(\begin{array}{cc}
A_{0}(s) & B_{0}(s) \\
-C_{0}(s) & D_{0}(s)
\end{array}\right)\left(\begin{array}{cc}
N(s) & Y(s) \\
0 & I
\end{array}\right)
\end{gathered}
$$

is a full equivalent transformation. Thus the following conditions are satisfied.

(a) According to the McMillan degree conditions of the full equivalent transformation (16) we have that

$$
\delta_{M}\left(\begin{array}{lll}
M(s) & 0 A_{0}(s) & B_{0}(s) \\
X(s) & I-C_{0}(s) & D_{0}(s)
\end{array}\right)=\delta_{M}\left(\begin{array}{cc}
A_{0}(s) & B_{0}(s) \\
-C_{0}(s) & D_{0}(s)
\end{array}\right)
$$

and

$$
\delta_{M}\left(\begin{array}{cc}
I & 0 \\
0 & R(s) \\
-N(s) & -Y(s) \\
0 & -I
\end{array}\right)=\delta_{M}\left(\begin{array}{cc}
I & 0 \\
0 & R(s)
\end{array}\right)
$$

In order for the second McMillan degree condition to be satisfied, $N(s)$ must be a constant marix (Pugh, 1976), i.e. $N(s)=N$. Taking into account that $N(s)$ is a constant matrix and the result from Pugh (1976) that constant matrices play no role in the McMillan degree condition, the relation (18) may be rewritten as

$$
\delta_{M}\left(\begin{array}{c}
R(s) \\
-Y(s)
\end{array}\right)=\delta_{M}(R(s)) .
$$


(b) The compound matrices

$$
Q(s)=\left(\begin{array}{cccc}
M(s) & 0 & A_{0}(s) & B_{0}(s) \\
X(s) & I & -C_{0}(s) & D_{0}(s)
\end{array}\right)
$$

and

$$
R(s)=\left(\begin{array}{cc}
I & 0 \\
0 & R(s) \\
-N(s) & -Y(s) \\
0 & -I
\end{array}\right)
$$

have no zeros in $C \cup\{\infty\}$. Equation (16) may be rewritten under an internal recoordination which does not change the internal structure of the equivalent matrices (Hayton et al., 1990) as

$$
\begin{aligned}
& \left(\begin{array}{cccc}
M(s) & 0 & A_{0}(s) & B_{0}(s) \\
X(s) & I & -C_{0}(s) & D_{0}(s)
\end{array}\right)\left(\begin{array}{cc}
I & 0 \\
0 & R(s) \\
-N & -Y(s) \\
0 & -I
\end{array}\right)=0 \Leftrightarrow \\
& \left(\begin{array}{cccc}
M(s) & 0 & A_{0}(s) & B_{0}(s) \\
X(s) & I & -C_{0}(s) & D_{0}(s)
\end{array}\right)\left(\begin{array}{cccc}
I & 0 & 0 & 0 \\
0 & I & 0 & 0 \\
-N & 0 & I & 0 \\
0 & 0 & 0 & I
\end{array}\right) \\
& \times\left(\begin{array}{cccc}
I & 0 & 0 & 0 \\
0 & I & 0 & 0 \\
N & 0 & I & 0 \\
0 & 0 & 0 & I
\end{array}\right)\left(\begin{array}{cc}
I & 0 \\
0 & R(s) \\
-N & -Y(s) \\
0 & -I
\end{array}\right)=0 \Leftrightarrow \\
& \left(\begin{array}{cccc}
M(s)-A_{0}(s) N & 0 & A_{0}(s) & B_{0}(s) \\
X(s)+C_{0}(s) N & I & -C_{0}(s) & D_{0}(s)
\end{array}\right)\left(\begin{array}{cc}
I & 0 \\
0 & R(s) \\
0 & -Y(s) \\
0 & -I
\end{array}\right)=0 \Leftrightarrow \\
& \left(\begin{array}{cc}
M(s)-A_{0}(s) N & 0 \\
X(s)+C_{0}(s) N & I
\end{array}\right)\left(\begin{array}{cc}
I & 0 \\
0 & R(s)
\end{array}\right) \\
& =\left(\begin{array}{cc}
A_{0}(s) & B_{0}(s) \\
-C_{0}(s) & D_{0}(s)
\end{array}\right)\left(\begin{array}{cc}
0 & Y(s) \\
0 & I
\end{array}\right) .
\end{aligned}
$$

Equating the terms $(1,1),(1,2),(2,1)$ and $(2,2)$ in $(19)$ we have that

$$
\begin{gathered}
(1,1) M(s)-A_{0}(s) N=0 \\
(1,2) A_{0}(s) Y(s)+B_{0}(s)=0 \Leftrightarrow Y(s)=-A_{0}(s)^{-1} B_{0}(s) \\
(2,1) X(s)+C_{0}(s) N=0 \\
(2,2) R(s)=-C_{0}(s) Y(s)+D_{0}(s) \\
\stackrel{(1,2)}{=} C_{0}(s) A_{0}(s)^{-1} B_{0}(s)+D_{0}(s) .
\end{gathered}
$$


Using the above equations we can now rewritte (19) as

$$
\begin{gathered}
\left(\begin{array}{ll}
0 & 0 \\
0 & I
\end{array}\right)\left(\begin{array}{cc}
I & 0 \\
0 & R(s)
\end{array}\right) \\
=\left(\begin{array}{cc}
A_{0}(s) & B_{0}(s) \\
-C_{0}(s) & D_{0}(s)
\end{array}\right)\left(\begin{array}{cc}
0 & -A_{0}(s)^{-1} B_{0}(s) \\
0 & I
\end{array}\right)
\end{gathered}
$$

and thus

$$
\left(\begin{array}{l}
0 \\
I
\end{array}\right) R(s)=\left(\begin{array}{cc}
A_{0}(s) & B_{0}(s) \\
-C_{0}(s) & D_{0}(s)
\end{array}\right)\left(\begin{array}{c}
-A_{0}(s)^{-1} B_{0}(s) \\
I
\end{array}\right)
$$

Equation (20) is a full equivalent transformation according to our assumption and, thus, it is easily seen that (21) is also a full equivalent transformation (just compare the compound matrices of the two transformations). Thus, the polynomial matrices of the ARRepresentations $\Sigma_{R}$ and $\Sigma_{G S}$ are full equivalent and therefore according to Theorem 4 the systems $\Sigma_{R}$ and $\Sigma_{G S}$ are fundamentally equivalent.

Using the symmetry property of (16) we can conclude using the same techniques that the symmetry transformation of (21) is the following:

$$
\left(\begin{array}{ll}
C_{0}(s) A_{0}(s)^{-1} & I
\end{array}\right)\left(\begin{array}{cc}
A_{0}(s) & B_{0}(s) \\
-C_{0}(s) & D_{0}(s)
\end{array}\right)=R(s)\left(\begin{array}{ll}
0 & I
\end{array}\right) .
$$

The bijective polynomial differential map which connects the behavioural space of the two systems is coming from the right transforming matrices of (21) and (22) according to Theorem 4 and is the following:

$$
w(t)=\left(\begin{array}{ll}
0 & I
\end{array}\right) \xi(t) \text { and } \xi(t)=\left(\begin{array}{c}
-A_{0}(\rho)^{-1} B_{0}(\rho) \\
I
\end{array}\right) w(t) .
$$

COROLlary 10 Note that the realization presented in Algorithm 1 is actually a subcase of the realization in (15), i.e. $A_{0}(\rho)=\rho E-A, B_{0}(\rho)=B, C_{0}(\rho)=C, D_{0}(\rho)=0$.

\section{Conclusions}

Fundamental equivalence between non-regular AR-Representations has been defined in Pugh et al. (1998) as the existence of a bijective polynomial differential map between the smooth and impulsive behavioural spaces of the equivalent systems, rather than a coincidence of these behavioural spaces (Kuijper, 1994). Based on this notion of equivalence we have proposed a new algorithm which reduces a full row rank ARRepresentation to a fundamental equivalent one in pencil form. An extension of this algorithm to a theoretical one has also been presented. The whole theory has been illustrated via an example.

\section{REFERENCES}

Hayton, G. E., Pugh, A. C. \& Fretwell, P. (1988) Infinite elementary divisors of a matrix polynomial and implications. Int. J. Control, 47, 53-64. 
Hayton, G. E., Walker, A. B. \& Pugh, A. C. (1990) Infinite frequency structure preserving transformations for general polynomial system matrices. Int. J. Control, 52, 1-14.

JANSSEN, P. H. (1988) General results on the McMillan degree and the Kronecker indices of ARMA and MFD models. Int. J. Control, 48, 591-608.

Karampetakis, N. P. \& Vardulakis, A. I. G. (1992) Matrix fractions and full system equivalence. IMA J. Math. Control Inform., 9, 147-160.

Karampetakis, N. P. \& VARdulakis, A. I. G. (1993) On the solution space of continuous time AR-representations. Proceedings of the 2nd European Control Conference, (ECC'93). pp. $1784-1789$.

KUIJPER, M. \& SHUMACHER, J. M. (1990) Realizations of autoregressive equations in pencil and descriptor form. SIAM J. Control Optimiz., 28, 1162-1189.

KuiJPer, M. (1994) First Order Representations of Linear Systems. Boston: Birkhauser.

Pugh, A. C. (1976) The McMillan degree of a polynomial system matrix. Int. J. Control, 24, 129135.

Pugh, A. C. \& Shelton, A. K. (1978) On a new definition of strict system equivalence. Int. J. Control, 27, 657-672.

Pugh, A. C., Antoniou, E., Karampetakis, N. P. \& Hayton, G. E. (1998) Fundamental equivalence of non-regular AR-representations. Mathematical Sciences Report, Number A315, Department of Mathematical Sciences, Loughborough University of Technology, UK.

Rosenbrock, H. H. (1970) State Space and Multivariable Theory. London: Nelson.

Shaohua, T. \& Joos, V. (1988) A singular system realization for arbitrary matrix fraction descriptions. ISCAS'88, 615-618.

VARdulakis, A. I. G. (1991) Linear Multivariable Control-Algebraic Analysis and Synthesis Methods. New York: Wiley.

Verghese, G. C., LeVy, B. \& Kailath, T. (1981) A generalized state space for singular systems. IEEE Trans. Autom. Control, 26, 811-831.

Willems, J. C. (1991) Paradigms and puzzles in the theory of dynamical systems. IEEE Trans. Autom. Control, 36, 259-294. 
